\title{
Oscillation of Second Order Nonlinear Neutral Differential Equations
}

\author{
Hussain Ali Mohamad ${ }^{1}$, Intidhar Zamil Mushtt ${ }^{2}$ \\ ${ }^{1}$ University of Baghdad, College of Science for Women, Baghdad, Iraq \\ ${ }^{2}$ Al Mustansiriyah University, College of Education, Baghdad, Iraq
}

Email address:

hussainmohamad22@gmail.com (H. A. Mohamad), intidharalbayaty@yahoo.com (I. Z. Mushtt)

\section{To cite this article:}

Hussain Ali Mohamad, Intidhar Zamil Mushtt. Oscillation of Second Order Nonlinear Neutral Differential Equations. Pure and Applied Mathematics Journal. Vol. 4, No. 2, 2015, pp. 62-65. doi: 10.11648/j.pamj.20150402.16

\begin{abstract}
The oscillation criteria are investigated for all solutions of second order nonlinear neutral delay differential equations. Our results extend and improve some results well known in the literature see ( [14] theorem 3.2.1 and theorem 3.2.2 pp.385-388). Some examples are given to illustrate our main results.
\end{abstract}

Keywords: Oscillation, Neutral Differential Equations

\section{Introduction}

In this paper we consider the second order neutral differential equations of the form

$[y(t)+\delta p(t) y(\tau(t))]^{\prime \prime}+q(t) f(y(\sigma(t)))=0, t \geq t_{0}, \delta= \pm 1(1.1)$

Under the following assumptions:

$\left(\mathrm{A}_{1}\right) p(t), q(t) \in C\left(\left[t_{0}, \infty\right) ; R^{+}\right), p(t)>0$.

$\left(\mathrm{A}_{2}\right) \tau(t), \sigma(t) \in C\left(\left[t_{0}, \infty\right) ; R\right), \lim _{t \rightarrow \infty} \tau(t)=\infty$,

$\lim _{t \rightarrow \infty} \sigma(t)=\infty, \tau(t)<t$ is increasing function. and $\tau^{k}(t)=\tau^{k-1}(\tau(t)), k=1,2, \ldots, n, \tau^{k-1}(t) \geq t_{0}$.

$\left(\mathrm{A}_{3}\right) f(\omega) \in C(R ; R), \omega f(\omega)>0$ for $\omega \neq 0, f(u v) \geq$ $f(u) f(v), u v>0$ and $|f(u)| \geq \beta|u|, \beta>0$.

By a solution of (1.1), we mean a function $y(t)$ such that $y(t)+\delta p(t) y(\tau(t))$ is twice continuously differentiable and $y(t)$ satisfies $(1.1)$ on $\left[t_{0}, \infty\right)$.

A solution is said to be oscillatory if it has arbitrarily large zeros, otherwise it is said to be nonoscillatory, that is a solution $y(t)$ is nonoscillatory if and only if $y(t)$ is eventually positive or eventually negative. Equation (1.1) is said to be oscillatory if every solution of (1.1) is oscillatory.

There has been a considerable investigation of the oscillations of second order neutral differential equations. For typical results we refer to the papers $[1,3,5-7,10,12-14]$. The results in this paper extend and improve some results well known in ([14], theorem 3.2.1 and theorem 3.2.2 pp.385-388). Also, we show that $\left(\mathrm{A}_{3}\right)$ implies that $f(t)$ is nondecreasing while in [13] they assume $\left(\mathrm{A}_{3}\right)$ holds in addition to $f(t)$ is nondecreasing. Some examples are given to illustrate the obtained results.

\section{Main Results}

In this section we present our main results, the following lemma shows that $\left(\mathrm{A}_{3}\right)$ implies $f(u)$ is nondecreasing.

Lemma 2.1 Suppose that $f \in C(R, R), u f(u) \geq 0$ for $u \neq 0, \frac{f(u)}{u} \geq \beta>0$ and $f(u v) \geq f(u) f(v)$ for $u v>0$. Then $f$ is nondecreasing function.

Proof: Assume that $u, v>0$ then $f(u), f(v)>0$. We have $f(u v) \geq f(u) f(v), f(u) \geq \beta u$.Then

$$
\begin{gathered}
f(u v) \geq \beta u f(v) \\
f(u v) \geq \beta v f(u) \\
0 \geq \beta[u f(v)-v f(u)] \\
v f(u) \geq u f(v) \\
\frac{v f(u)}{u f(u)} \geq \frac{u f(v)}{u f(u)} \\
\frac{f(v)}{f(u)} \leq \frac{v}{u}
\end{gathered}
$$

If $v \leq u$ then (2.1) implies that $f(v) \leq f(u)$

Hence $f$ is nondecreasing function.

Lemma 2.2. [7] Suppose that $f(t) ; g(t): R^{+} \rightarrow R^{+}$; $g(t)<t, \lim _{t \rightarrow \infty} g(t)=\infty$ and 


$$
\lim \inf _{t \rightarrow \infty} \int_{g(t)}^{t} f(s) d s>\frac{1}{e}
$$

Then the inequality $y^{\prime}(t)+f(t) y(g(t)) \leq 0$ cannot has eventually positive solution, and the inequality $y^{\prime}(t)+$ $f(t) y(g(t)) \geq 0$ cannot has eventually negative solution.

In the following theorem we investigate eq. (1.1) when $\delta=1$.

Theorem 2.3 Assume that $\left(A_{1}\right)-\left(A_{3}\right)$ hold, $\sigma(t) \geq t$, and

$$
\lim \sup _{t \rightarrow \infty} \sum_{i=1}^{\frac{n+1}{2}} \prod_{k=1}^{2 i-1} p\left(\tau^{k-1}(\sigma(t))\right)<1
$$

For some positive odd integer $n$ and there exists a function $\left.h(t) \epsilon C^{1}\left[t_{0}, \infty\right), R^{+}\right]$such that

$\lim \sup _{t \rightarrow \infty} \int_{T}^{t}\left[\beta h(s) q(s) f(\gamma(s))-\frac{\left[h^{\prime}(s)\right]^{2}}{4 h(s)}\right] d s=\infty, T \geq t_{0}$

where $\gamma(t)=1-\sum_{i=1}^{\frac{n+1}{2}} \prod_{k=1}^{2 i-1} p\left(\tau^{k-1}(\sigma(t))\right)$. Then every solution of (1.1) oscillates.

Proof. Assume for the sake of contradiction that (1.1) has a nonoscillatory solution, let $y(t)$ be eventually positive solution of (1.1) and there exists a positive odd integer $n$ such that $y\left(\tau^{i}(t)\right)>0, i=0,1,2, \ldots, n, y(\sigma(t))>0$, for $t \geq t_{1} \geq t_{0}$. Let

$$
z(t)=y(t)+p(t) y(\tau(t))
$$

From (1.1) and (2.4) it follows that

$$
z^{\prime \prime}(t)=-q(t) f(y(\sigma(t))) \leq 0, t \geq t_{1}
$$

Since $z(t)>0$ then it follows that $z^{\prime}(t)>0, t \geq t_{2} \geq t_{1}$ Form (2.4) we obtain

$$
\begin{aligned}
& y(t)=z(t)-p(t) y(\tau(t)) \\
& =z(t)-p(t)\left[z(\tau(t))-p(\tau(t)) y\left(\tau^{2}(t)\right)\right] \\
& =z(t)-p(t)\left[z(\tau(t))-p(\tau(t))\left[z\left(\tau^{2}(t)\right)-p\left(\tau^{2}(t)\right) y\left(\tau^{3}(t)\right)\right]\right] \\
& = \\
& z(t)+\prod_{i=0}^{n} p\left(\tau^{i}(t)\right) y\left(\tau^{n+1}(t)\right)+ \\
& \sum_{i=1}^{\frac{n-1}{2}} \prod_{k=0}^{2 i-1} p\left(\tau^{k}(t)\right) z\left(\tau^{2 i}(t)\right)- \\
& \sum_{i=1}^{\frac{n+1}{2}} \prod_{k=1}^{2 i-1} p\left(\tau^{k-1}(t)\right) z\left(\tau^{2 i-1}(t)\right) \\
& y(t) \geq z(t)-\sum_{i=1}^{\frac{n+1}{2}} \prod_{k=1}^{2 i-1} p\left(\tau^{k-1}(t)\right) z\left(\tau^{2 i-1}(t)\right) \\
& \geq\left[1-\sum_{i=1}^{\frac{n+1}{2}} \prod_{k=1}^{2 i-1} p\left(\tau^{k-1}(t)\right)\right] z(t)
\end{aligned}
$$$$
y(\sigma(t)) \geq\left[1-\sum_{i=1}^{\frac{n+1}{2}} \prod_{k=1}^{2 i-1} p\left(\tau^{k-1}(\sigma(t))\right)\right] z(\sigma(t))
$$

By $\left(\mathrm{A}_{3}\right)$ and Lemma 2.1 we get

$$
\begin{array}{r}
f(y(\sigma(t))) \geq f\left(1-\sum_{i=1}^{\frac{n+1}{2}} \prod_{k=1}^{2 i-1} p\left(\tau^{k-1}(\sigma(\mathrm{t}))\right) z(\sigma(\mathrm{t}))\right) \\
\geq f\left(1-\sum_{i=1}^{\frac{n+1}{2}} \prod_{k=1}^{2 i-1} p\left(\tau^{k-1}(\sigma(\mathrm{t}))\right) f(z(\sigma(\mathrm{t})))\right.
\end{array}
$$

$$
f(y(\sigma(t))) \geq f\left(1-\sum_{i=1}^{\frac{n+1}{2}} \prod_{k=1}^{2 i-1} p\left(\tau^{k-1}(\sigma(\mathrm{t}))\right) \beta z(\sigma(\mathrm{t}))\right.
$$

Substituting (2.7) into (2.5) we obtain $z^{\prime \prime}(t)+$

$$
\beta q(t)\left[f\left(1-\sum_{i=1}^{\frac{n+1}{2}} \prod_{k=1}^{2 i-1} p\left(\tau^{k-1}(\sigma(\mathrm{t}))\right)\right)\right] z(\sigma(t)) \leq 0
$$

Define

$$
w(t)=\frac{h(t) z^{\prime}(t)}{z(\sigma(t))}
$$

It is obvious that $w(t)>0$. Let $\gamma(t)=1-\sum_{i=1}^{\frac{n+1}{2}} \prod_{k=1}^{2 i-1} p\left(\tau^{k-1}(\sigma(t))\right)$ then differentiate (2.9) and using (2.8) it follows that

$$
\begin{gathered}
\frac{h(t) w^{\prime}(t)-w(t) h^{\prime}(t)}{(h(t))^{2}} z(t)+\frac{w(t)}{h(t)} z^{\prime}(t)+\beta q(t) f(\gamma(t)) z(\sigma(t)) \leq 0(2.8) \\
w^{\prime}(t)-\frac{h^{\prime}(t)}{h(t)} w(t)+\frac{z^{\prime}(t)}{z(t)} w(t)+\beta h(t) q(t) f(\gamma(t)) \leq 0
\end{gathered}
$$

Then for $t \geq t_{3}(=T) \geq t_{2}$

$$
\begin{aligned}
& w^{\prime}(t) \leq \frac{h^{\prime}(t)}{h(t)} w(t)-\frac{z^{\prime}(t)}{z(t)} w(t)-\beta h(t) q(t) f(\gamma(t)) \\
& =\frac{h^{\prime}(t)}{h(t)} w(t)-\frac{1}{h(t)} w^{2}(t)-\beta h(t) q(t) f(\gamma(t)) \\
& =-\beta h(t) q(t) f(\gamma(t))-\left[\frac{1}{\sqrt{h(t)}} w(t)-\frac{h^{\prime}(t)}{2 \sqrt{h(t)}}\right]^{2}+\frac{\left(h^{\prime}(t)\right)^{2}}{4 h(t)} \\
& \leq-\beta h(t) q(t) f(\gamma(t))+\frac{\left(h^{\prime}(t)\right)^{2}}{4 h(t)} \\
& w^{\prime}(t) \leq-\left[\beta h(t) q(t) f(\gamma(t))-\frac{\left(h^{\prime}(t)\right)^{2}}{4 h(t)}\right]
\end{aligned}
$$

Integrating the last inequality from $T$ to $t$, we get

$$
w(t) \leq w(T)-\int_{T}^{t}\left[\beta h(s) q(s) f(\gamma(s))-\frac{\left(\mathrm{h}^{\prime}(s)\right)^{2}}{4 h(s)}\right] d s,
$$

as $t \rightarrow \infty$ the last inequality leads to $w(t) \rightarrow-\infty$, which is a contradiction.

Example 1 Consider the neutral differential equation

$$
\begin{aligned}
& {\left[y(t)+e^{-t} y\left(t-\frac{3 \pi}{2}\right)\right]^{\prime \prime}+e^{-\frac{\pi}{2}} f(y(t+2 \pi))=0, t \geq 1,} \\
& \tau(t)=t-\frac{3 \pi}{2}, \sigma(t)=t+2 \pi, p(t)=e^{-t}, \\
& f(y(t+2 \pi))=y(t+2 \pi)\left[e^{\frac{\pi}{2}}-2 e^{-t+\frac{\pi}{2}}\right], q(t)=e^{-\frac{\pi}{2}}
\end{aligned}
$$

one can find that all conditions of theorem 2.3 are hold

Let $n=3, \beta=3, h(t)=c>0$,

$\lim \sup _{n \rightarrow \infty} \sum_{i=1}^{\frac{n+1}{2}} \prod_{k=1}^{2 i-1} p\left(\tau^{k-1}(\sigma(t))\right)=$ $=\lim _{t \rightarrow \infty}\left[e^{-t-2 \pi}+e^{-3 t-2 \pi}\right]=0$ 
$\lim \sup _{t \rightarrow \infty} \int_{T}^{t}\left[\beta h(s) q(s) f(\gamma(s))-\frac{\left[h^{\prime}(s)\right]^{2}}{4 \alpha h(s)}\right] d s=$

$=\lim _{t \rightarrow \infty} \int_{T}^{t} \frac{3 c}{2} e^{-\frac{\pi}{2}} f(\gamma(s)) d s=\infty$

so every solution of eq.(E1) oscillates, for instance $y(t)=$ $\sin t$ is such solution.

The following theorem investigate equation (1.1) when $\delta=-1$.

Theorem 2.4 Assume $\left(A_{1}\right)-\left(A_{3}\right)$ hold, $p(t) \leq 1$, $\tau^{n}(\sigma(t)) \geq t, \tau^{-n}(\sigma(\alpha(t))) \leq t, \alpha(t)>t$ and there exists a function $\left.h(t) \in C^{1}\left[t_{0}, \infty\right), R^{+}\right]$such that

$$
\int_{T}^{\infty}\left[\beta h(s) q(s) f\left(\gamma_{1}(s)\right)-\frac{\left(h^{\prime}(s)\right)^{2}}{4 h(s)} d s\right]=\infty, T \geq t_{0}
$$

$\liminf _{t \rightarrow \infty} \int_{\tau^{-n}(\sigma(\alpha(t)))}^{t} \int_{s}^{\alpha(s)} q(v) f(\theta(v)) d v d s>\frac{1}{\beta e}$

where $\quad \gamma_{1}(t)=1+\sum_{i=1}^{n} \prod_{k=0}^{i-1} p\left(\tau^{k}(\sigma(t))\right), \theta(t)=$ $\sum_{i=1}^{n} \prod_{k=0}^{i-1} \frac{1}{p\left(\tau^{-k}(\sigma(t))\right)}$. Then every solution of $(1.1)$ is oscillatory.

Proof: Assume for the sake of contradiction that (1.1) has a eventually positive solution $y(t)$, then there exists $t_{1} \geq t_{0}$ such that for $t \geq t_{1}, y\left(\tau^{i}(t)\right)>0, i=1,2, \ldots, n$, where $n$ is positive integer and $y(\sigma(t))>0$. Let

$$
z(t)=y(t)-p(t) y(\tau(t)
$$

From equation (1.1) and (2.13) we get

$$
z^{\prime \prime}(t)=-q(t) f(y(\sigma(t))) \leq 0, t \geq t_{1}
$$

hence $z^{\prime}(t), z(t)$ are monotone functions, we claim that $z^{\prime}(t)>0$, for $t \geq t_{2} \geq t_{1}$ otherwise $z^{\prime}(t)<0$ leads to $z(t)<0$ for $t \geq t_{2} \geq t_{1}$ and $\lim _{t \rightarrow \infty} z(t)=-\infty$ it follows that $y(t)<p(t) y(\tau(t)) \leq y(\tau(t))$ then $y(t)$ is bounded.

On the other hand $z(t)>-p(t) y(\tau(t) \geq-y(\tau(t))$

Then $\lim _{t \rightarrow \infty} y(t)=\infty$, leads to a contradiction. Therefore $z^{\prime}(t)>0$ for $t \geq t_{2} \geq t_{1}$, there are two cases for $z(t)$ :

(a) $z(t)>0$ for $t \geq t_{2} \geq t_{1}$; (b) $z(t)<0$ for $t \geq t_{2} \geq t_{1}$

Case (a) $z(t)>0, z^{\prime}(t)>0$ and $z^{\prime \prime}(t) \leq 0$

From (2.13) it follows that

$$
\begin{gathered}
y(t)=z(t)+p(t) y(\tau(t))= \\
=z(t)+p(t)\left[z(\tau(t))+p(\tau(t)) y\left(\tau^{2}(t)\right)\right] \\
=z(t)+p(t) z(\tau(t))+p(t) p(\tau(t))\left[z\left(\tau^{2}(t)\right)\right. \\
\left.+p\left(\tau^{2}(t)\right) y\left(\tau^{3}(t)\right)\right]
\end{gathered}
$$

$y(t)=$

$z(t)+\sum_{i=1}^{n} \prod_{k=0}^{i-1} p\left(\tau^{k}(t)\right) z\left(\tau^{i}(t)\right)+\prod_{i=0}^{n} p\left(\tau^{i}(t)\right) y\left(\tau^{n+1}(t)\right)$

$$
\begin{gathered}
\geq z(t)+\sum_{i=1}^{n} \prod_{k=0}^{i-1} p\left(\tau^{k}(t)\right) z\left(\tau^{i}(t)\right) \\
\geq\left[1+\sum_{i=1}^{n} \prod_{k=0}^{i-1} p\left(\tau^{k}(t)\right)\right] z\left(\tau^{n}(t)\right)
\end{gathered}
$$

Then there exists $t_{3}(=T) \geq t_{2}$ such that for $t \geq T$

$$
y(\sigma(t)) \geq\left[1+\sum_{i=1}^{n} \prod_{k=0}^{i-1} p\left(\tau^{k}(\sigma(t))\right)\right] z\left(\tau^{n}(\sigma(t))\right)
$$

Then by using $\left(A_{3}\right)$ and Lemma 2.1 we have

$f(y(\sigma(t))) \geq f\left[1+\sum_{i=1}^{n} \prod_{k=0}^{i-1} p\left(\tau^{k}(\sigma(t))\right)\right] f\left(z\left(\tau^{n}(\sigma(t))\right)\right)$

$$
\geq \beta f\left(1+\sum_{i=1}^{n} \prod_{k=0}^{i-1} p\left(\tau^{k}(\sigma(t))\right)\right) z\left(\tau^{n}(\sigma(t))\right)
$$

By substituting (2.16) in (1.1) we obtain

$z^{\prime \prime}(t)+\beta q(t) f\left(1+\sum_{i=1}^{n} \prod_{k=0}^{i-1} p\left(\tau^{k}(\sigma(t))\right)\right) z\left(\tau^{n}(\sigma(t))\right) \leq 0$

Define

$$
w(t)=\frac{h(t) z^{\prime}(t)}{z(t)}
$$

Then $w(t)>0$, moreover

$$
\begin{aligned}
& w^{\prime}(t)+\frac{z^{\prime}(t)}{z(t)} w(t)-\frac{h^{\prime}(t)}{h(t)} w(t)+ \\
& \beta h(t) q(t) f\left(\gamma_{1}(t)\right) \frac{z\left(\tau^{n}(\sigma(t))\right)}{z(t)} \leq 0
\end{aligned}
$$

Where $\gamma_{1}(t)=1+\sum_{i=1}^{n} \prod_{k=0}^{i-1} p\left(\tau^{k}(\sigma(t))\right)$.

Since $z(t) \leq z\left(\tau^{n}(\sigma(t))\right)$ then

$$
\begin{array}{r}
w^{\prime}(t) \leq-\frac{z^{\prime}(t)}{z(t)} w(t)+\frac{h^{\prime}(t)}{h(t)} w(t)-\beta h(t) q(t) f\left(\gamma_{1}(t)\right) \\
=-\frac{1}{h(t)} w^{2}(t)+\frac{h^{\prime}(t)}{h(t)} w(t)-\beta h(t) q(t) f\left(\gamma_{1}(t)\right) \\
=-\left[\frac{1}{\sqrt{h(t)}} w(t)-\frac{h^{\prime}(t)}{2 \sqrt{h(t)}}\right]^{2}+\frac{\left(h^{\prime}(t)\right)^{2}}{4 h(t)} \\
-\beta h(t) q(t) f\left(\gamma_{1}(t)\right)
\end{array}
$$

Thus

$$
w^{\prime}(t) \leq \frac{\left(h^{\prime}(t)\right)^{2}}{4 h(t)}-\beta h(t) q(t) f\left(\gamma_{1}(t)\right)
$$

Integrating the last inequality from $T$ to $t$ we get

$$
w(t) \leq w(T)-\int_{T}^{t}\left[\beta h(s) q(s) f\left(\gamma_{1}(s)-\frac{\left(h^{\prime}(s)\right)^{2}}{4 h(s)}\right] d s\right.
$$

as $t \rightarrow \infty$, and by (2.11) we deduce that $w(t) \rightarrow-\infty$ this is a contradiction.

Case (b) $z(t)<0, z^{\prime}(t)>0$ and $z^{\prime \prime}(t) \leq 0$

From (2.13) it follows that $y(\tau(t))=\frac{1}{p(t)}(y(t)-z(t))$

Then $y(t)=\frac{1}{p\left(\tau^{-1}(t)\right)} y\left(\tau^{-1}(t)\right)-\frac{1}{p\left(\tau^{-1}(t)\right)} z\left(\tau^{-1}(t)\right)$

$y(t)=\frac{1}{p\left(\tau^{-1}(t)\right)}\left[\frac{1}{p\left(\tau^{-2}(t)\right.} y\left(\tau^{-2}(t)\right)\right.$

$\left.-\frac{1}{p\left(\tau^{-2}(t)\right)} z\left(\tau^{-2}(t)\right)\right]-\frac{1}{p\left(\tau^{-1}(t)\right)} z\left(\tau^{-1}(t)\right)$

$y(t)=\frac{1}{\prod_{i=1}^{n} p\left(\tau^{-i}(t)\right)} y\left(\tau^{-n}(t)\right)-\sum_{i=1}^{n} \prod_{k=1}^{i} \frac{1}{p\left(\tau^{-k}(t)\right)} z\left(\tau^{-i}(t)\right)$

$\geq-\sum_{i=1}^{n} \prod_{k=1}^{i} \frac{1}{p\left(\tau^{-k}(t)\right)} z\left(\tau^{-i}(t)\right)$ 
$\geq-\sum_{i=1}^{n} \prod_{k=1}^{i} \frac{1}{p\left(\tau^{-k}(t)\right)} Z\left(\tau^{-n}(t)\right)$

Then there exists $t_{3}(=T) \geq t_{2}$ such that for $t \geq T$

$$
y(\sigma(t)) \geq-\sum_{i=1}^{n} \prod_{k=1}^{i} \frac{1}{p\left(\tau^{-k}(\sigma(t))\right)} z\left(\tau^{-n}(\sigma(t))\right)
$$

Then by using $\left(A_{3}\right)$ and Lemma 2.1 we have

$$
\begin{gathered}
f(y(\sigma(t))) \geq f\left(\sum_{i=1}^{n} \prod_{k=1}^{i} \frac{1}{p\left(\tau^{-k}(\sigma(t))\right)}\right) f\left(-z\left(\tau^{-n}(\sigma(t))\right)\right) \\
\geq-\beta f\left(\sum_{i=1}^{n} \prod_{k=1}^{i} \frac{1}{p\left(\tau^{-k}(\sigma(t))\right)}\right) z\left(\tau^{-n}(\sigma(t))\right)
\end{gathered}
$$

By substituting (2.19) in (2.14) we obtain

$$
z^{\prime \prime}(t)-\beta q(t) f\left(\sum_{i=1}^{n} \prod_{k=1}^{i} \frac{1}{p\left(\tau^{-k}(\sigma(t))\right)}\right) z\left(\tau^{-n}(\sigma(t))\right) \leq 0
$$

Integrating (2.20) from $\mathrm{t}$ to $\alpha(t)$ where $\alpha(t)>t$ is continuous function, we get

$$
z^{\prime}(t)+\beta z\left(\tau^{-n}(\sigma(\alpha(t)))\right) \int_{t}^{\alpha(t)} q(s) f(\theta(s)) \geq 0
$$

where $\theta(t)=\sum_{i=1}^{n} \prod_{k=0}^{i-1} \frac{1}{p\left(\tau^{-k}(\sigma(t))\right)}$. By lemma 2.2 and condition (2.13) it follows that eq(2.21) cannot has eventually negative solution, which is a contradiction.

Then every solutions of (1.1) is oscillatory.

Example 2 Consider the neutral differential equation

$$
\begin{aligned}
& {\left[y(t)-e^{-2 \pi}(3-\sin t) y(t-2 \pi)\right]^{\prime \prime}} \\
& \quad+2 e^{-\frac{9 \pi}{2}}(\cos t+2) y\left(t-\frac{9 \pi}{2}\right)=0, t \geq T
\end{aligned}
$$

one can find that all conditions of theorem 2.4(or corollary 2.5 ) are hold, to see that:

$$
\begin{aligned}
& \text { Let } n=2, \beta=\frac{1}{2}, h(t)=c>0, \\
& f\left(\gamma_{1}(t)\right)=1+e^{-2 \pi}(3+\cos t)+e^{-2 \pi}(3+\cos t)^{2} \\
& f(\theta(t))=\frac{1}{e^{-2 \pi}(3+\cos t)}+\frac{1}{e^{-4 \pi}(3+\cos t)^{2}} \\
& \lim _{t \rightarrow \infty} \int_{T}^{t} c e^{-\frac{9 \pi}{2}}(\cos s+2) f\left(\gamma_{1}(s)\right) d s=\infty \\
& \lim _{t \rightarrow \infty} \int_{T}^{t} c e^{-\frac{9 \pi}{2}}(\cos s+2) f(\theta(s)) d s=\infty
\end{aligned}
$$

so every solution of eq.(E2) oscillate for instance $y(t)=$ $e^{t} \cos t$ is such a solution.

\section{Conclusions}

As mention in the abstract the results in this paper extended and improved theorem 3.2.1 and theorem 3.2.2 [4]

The authors in[13] assume $\left(\mathrm{A}_{3}\right)$ holds in addition to $f(u)$ is nondecreasing while we show that $\left(\mathrm{A}_{3}\right)$ implies $f(u)$ is nondecreasing. Moreover we can establish other results by assuming $f(u v) \leq f(u) f(v)$ and $f(u) \leq \beta u$ also implies that $f(u)$ is nondecreasing in similar way.

\section{References}

[1] R. P. Agarwal, M. Bohner, T. Li, C. Zhang; A new approach in the study of oscillatory behavior of even-order neutral delay differential equations. Appl. Math. Comput., 225 (2013),787794.

[2] R. Al-Hamouri and A. Zein: Oscillation Criteria for Certain even order neutral Delay Differential Equations, international Journal of Differential Equations, Vol 2014, Article ID 437278,5 pages.

[3] B. Baculikov_a, J. D_zurina; Oscillation criteria for second order neutral differential equations. Mathematica Bohemica, 125 (2000) 2 145-153.

[4] J. G. Dix Oscillation of solutions to a neutral di_erential equation involving an n-order operator with variable coefficients and a forcing term.. Differ. Equ. Dyn. Syst., 22 (2014), $15\{31$.

[5] M. Hasanbulli, Yu. V. Rogovchenko; Oscillation criteria for second order nonlinear neutral differential equations. Appl. Math. Comput., 215 (2010) 4392-4399.

[6] B. Karpuz, Ö. Öcalan, S. Özturk; Comparison theorems on the oscillation and asymptotic behaviour of higher-order neutral differential equations. Glasgow Math. J., 52 (2010) 107-114.

[7] G.S. Ladde, V. Lakshmikantham, B.G. Zhang : Oscillation Theory of Differential Equations with deviating Arguments Marcel Dekker inc, New York and Basel.,(1987).

[8] Tongxing Li, Blanka Baculkov and Jozef Durina; Oscillatory behavior of second-order nonlinear neutral differential equations with distributed deviating arguments. Boundary value problem, 2014 (2014) 68 1-15.

[9] T. Li; Comparison theorems for second-order neutral differential equations of mixed type. Electro. J. Diff. Equ., 2010 (2010) no. 167, 1-7.

[10] T. Li, R. P. Agarwal, M. Bohner; Some oscillation results for second-order neutral differential equations. J. Indian Math. Soc., 79 (2012) 97-106.

[11] L. Li, F. Meng; New results on Oscillation for even order neutral differential equations with deviating arguments. Advance Pure Mathematic, 1 (2011) 49-53.

[12] T. Li, Z. Han, P. Zhao and S. Sun; Oscillation of even order differential equations. Advance in Difference Equations, 2010 (2010) 1-9.

[13] R. N. Rath, L. N. Padhy and N. Misra; Oscillation of solution of nonlinear neutral delay differential equations of higher order for $p(t)= \pm 1$. Archivum Mathematicum 40 (2004)359366.

[14] Samir Saker; Oscillation Theory of delay differential and di_erence equations of second order. VDM Verlag Dr. Muller, 2010 .

[15] M. K. Yildiz; Oscillation results of higher order nonlinear neutral delay differential equations with oscillating coefficients. Advance in Dynamical system and application, Vol3 No2 (2008)297-303. 